\title{
The Thoracic Surgery Social Media Network: Early experience and lessons learned
}

Jessica G. Y. Luc, MD, ${ }^{a}$ Maral Ouzounian, MD, PhD, ${ }^{b}$ Edward M. Bender, MD, ${ }^{c}$ Arie Blitz, MD, MBA, ${ }^{d}$ Nikki L. Stamp, MBBS(Hons), FRACS, ${ }^{\mathrm{e}}$ Thomas K. Varghese, Jr, MD, MS, ${ }^{\mathrm{f}}$ David T. Cooke, MD, ${ }^{\mathrm{g}}$ and Mara B. Antonoff, $\mathrm{MD}^{\mathrm{h}}$

\section{ABSTRACT}

Background: The Thoracic Surgery Social Media Network (TSSMN) is a social media collaborative formed in 2015 by The Annals of Thoracic Surgery and The Journal of Thoracic and Cardiovascular Surgery to bring social media attention to key publications from both journals and to highlight major accomplishments in the specialty. Our aim is to describe TSSMN's preliminary experience and lessons learned.

Methods: Twitter analytics was used to obtain information regarding the @ TSSMN Twitter handle and \#TSSMN hashtag. TweetChat and general hashtag \#TSSMN analytics were measured using Symplur (Symplur LLC, Los Angeles, Calif). A TSSMN Tweeter App was created, and its use and downloads were analyzed.

Results: Hashtag \#TSSMN has a total of 17,181 tweets, 2100 users, and $32,226,280$ impressions, with peaks in tweeting activity corresponding to TweetChats. Thirteen 1-hour TweetChats drew a total of 489 participants, 5195 total tweets, and 17,297,708 total impressions. The top demographic category of TweetChat participants included Doctors (47\%), Advocates/Supports (11\%), and Unknown (10\%), with 3\% characterized as patients. The TSSMN Tweeter iTunes App (Apple, Cupertino, Calif) was downloaded 3319 times with global representation. A total of 859 articles were viewed through the App, with 450 articles from The Annals of Thoracic Surgery and 409 from The Journal of Thoracic and Cardiovascular Surgery.

Conclusions: We demonstrate that TSSMN further enhances the ability for the journals to connect with their readership and the cardiothoracic community. Ongoing studies to correlate social media attention with article reads, articlelevel metrics, citations, and journal impact factor are eagerly awaited. (J Thorac Cardiovasc Surg 2019;158:1127-36)

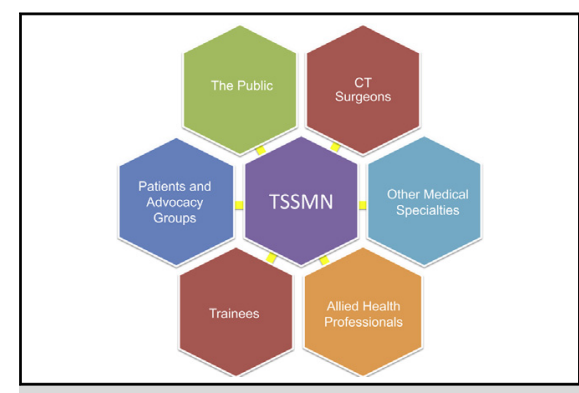

The TSSMN connects a wide community of readers.

Central Message

Thoracic Surgery Social Media Network (TSSMN) is a collaboration of The Annals of Thoracic Surgery and The Journal of Thoracic and Cardiovascular Surgery to bring published articles to social media.

Perspective

Thoracic Surgery Social Media Network (TSSMN) tweeting activity has enhanced the ability of authors, readers, and the public to interact regarding key publications. Ongoing studies correlating article dissemination via social media with objective measures of article reads and citations are eagerly awaited.

See Commentary on page 1137.
The Thoracic Surgery Social Media Network (TSSMN) is a social media collaborative formed in 2015 by The Annals of Thoracic Surgery and The Journal of Thoracic and Cardiovascular Surgery. ${ }^{1}$ Delegates selected for this initiative are key members of the cardiothoracic surgery community and innovators in social media (Table 1). Delegates represent adult cardiac, congenital, and general thoracic surgery subspecialties and have broad geographic representation.

\footnotetext{
From the a Division of Cardiovascular Surgery, Department of Surgery, University of British Columbia, Vancouver, British Columbia, Canada; ${ }^{\mathrm{b}}$ Division of Cardiovascular Surgery, Peter Munk Cardiac Centre, Toronto General Hospital, University of Toronto, Toronto, Ontario, Canada; ' Department of Cardiothoracic Surgery, Stanford University, Palo Alto, Calif; ${ }^{\mathrm{d}}$ Division of Cardiac Surgery, University of Cincinnati Medical Center, Cincinnati, Ohio; ${ }^{\mathrm{e} D e p a r t m e n t ~ o f ~ C a r d i o t h o r a c i c ~ S u r g e r y ~}$ \& Transplantation, Fiona Stanley Hospital, Murdoch, Australia; ${ }^{\mathrm{f}}$ Division of Cardiothoracic Surgery, Department of Surgery, University of Utah, Salt Lake City, Utah; ${ }^{\mathrm{g}}$ Section of General Thoracic Surgery, University of California Davis Health, Sacramento, Calif; and ${ }^{\mathrm{h}}$ Department of Thoracic and Cardiovascular Surgery, University of Texas MD Anderson Cancer Center, Houston, Tex.
}

This article has been copublished in The Annals of Thoracic Surgery and The Journal of Thoracic and Cardiovascular Surgery.

Received for publication March 5, 2019; revisions received May 28, 2019; accepted for publication June 3, 2019; available ahead of print Aug 15, 2019.

Address for reprints: Mara B. Antonoff, MD, Thoracic and Cardiovascular Surgery, 1400 Pressler St, Unit 1489, Houston, TX 77030 (E-mail: mbantonoff@ mdanderson.org).

$0022-5223 / \$ 36.00$

(C) 2019 by The Society of Thoracic Surgeons and The American Association for Thoracic Surgery. Published by Elsevier Inc.

https://doi.org/10.1016/j.jtcvs.2019.06.099 


\section{Abbreviations and Acronyms \\ TSSMN $=$ Thoracic Surgery Social Media Network}

Trainee members were invited to join in 2017 and provide a unique perspective on issues related to training, mentorship, and transitioning to independent practice.

Each month, TSSMN delegates disseminate new, original research published in cardiothoracic surgery across Twitter. Delegates provide commentary contextualizing the recently published papers within our respective areas and highlight the potential impact a study may have on our surgical community. Tweets are accompanied with the hashtag \#TSSMN, have links to the selected article, and are retweeted by other delegates and the @TSSMN account for broad dissemination.

Articles are chosen that are provocative, feature innovative techniques, or simply because they are of interest to the delegate. Often, featured articles that are open access (ie, full-text is available to the public) are chosen for dissemination. The TSSMN tweets serve to stimulate a discussion around the main findings of the article, applications to clinical practice, limitations, and unanswered questions through broad engagement of the social media community. The target audience for TSSMN activities includes other scientists and clinicians (surgeons, cardiologists, pulmonologists, oncologists, etc), as well as the lay public and patients.

In addition to direct tweeting of articles, members of TSSMN regularly engage in live tweeting from our national scientific sessions. By using hashtags, such as \#AATS2019 (the American Association for Thoracic Surgery Annual Meeting) and \#STS2019 (The Society of Thoracic Surgeons Annual Meeting), delegates provide minute-to-minute updates regarding conference events, keynote speakers, and the latest research in our field. ${ }^{2}$

Furthermore, several times a year, members of TSSMN host 1-hour live TweetChats addressing interesting topics with relevant papers from both journals. ${ }^{3}$ The moderator of the TweetChat prepares questions for discussion, and the session serves as a journal club for cardiothoracic trainees and surgeons with global participation. Authors of the selected papers are invited to participate in the chat, providing a forum for high-level interaction with interested readers and an in-depth exploration of the papers' content.

The present report describes the preliminary experience of the TSSMN group along with lessons learned.

\section{MATERIAL AND METHODS}

\section{The Thoracic Surgery Social Media Network}

The TSSMN was formed by creation of a Twitter handle @TSSMN and hashtag \#TSSMN to link conversations of cardiothoracic surgical literature
TABLE 1. Current thoracic surgery social media delegates, 2019

\begin{tabular}{|c|c|c|}
\hline Name & Twitter handle & Country \\
\hline $\begin{array}{c}\text { Thoracic Surgery Social } \\
\text { Media Network }\end{array}$ & @TSSMN & \\
\hline \multicolumn{3}{|l|}{ Adult cardiac surgery } \\
\hline Rakesh Arora & @TheRakeshArora & Canada \\
\hline Edward Bender & @ebender001 & USA \\
\hline Arie Blitz & @ArieBlitzMD & USA \\
\hline Maral Ouzounian & @OuzounianMD & Canada \\
\hline Nikki Stamp & @ drnikkistamp & Australia \\
\hline \multicolumn{3}{|l|}{ General thoracic surgery } \\
\hline Mara Antonoff & @maraantonoff & USA \\
\hline David Cooke & @DavidCookeMD & USA \\
\hline Biniam Kidane & @biniamkidaneMD & Canada \\
\hline Tom Varghese & @TomVargheseJr & USA \\
\hline \multicolumn{3}{|l|}{ Congenital cardiac surgery } \\
\hline Matthew Liava'a & @DrM_Liavaa & USA \\
\hline \multicolumn{3}{|l|}{ Trainee representatives } \\
\hline Michael Archer & @ archerm2 & USA \\
\hline Caitlin Harrington & @CHarringtonMD & USA \\
\hline Jessica Luc & @ JessicaLuc1 & Canada \\
\hline Tamara Nihici & @tamaranihici & United Kingdom \\
\hline
\end{tabular}

USA, United States of America.

to the handle. An information page regarding TSSMN as well as the handles of TSSMN delegates were promoted on The Annals of Thoracic Surgery and The Journal of Thoracic and Cardiovascular Surgery journal websites (Table 1). Twitter analytics was used to obtain information about the performance of the @TSSMN Twitter handle and \#TSSMN hashtag.

\section{Definitions}

Definitions of Twitter nomenclature have been reviewed by Luc and colleagues. $^{2,3}$ In brief, a handle is a Twitter account with a corresponding username. Hashtags identify specific topics and allow one to connect with other people tweeting or interested in similar subjects and are the primary modality by which tweets in a given campaign or TweetChat are connected. Hashtags are "clickable" as well, meaning that when a hashtag is tapped, it will populate a list of all tweets containing that hashtag and thus related to that topic. Impressions are the number of times article tweets were viewed by unique individuals. Followers are the people following an account, which means that they allow your tweets to be automatically populated in their Twitter feed, which is a live stream of all content being posted by the individuals they are following.

\section{TweetChats}

TSSMN was created in May 2015. Thirteen TweetChats were held between September 17, 2015, and February 19, 2019, and were analyzed as part of this study. TweetChats were 1-hour in length and were scheduled occurrences with broad advertisement by TSSMN, TSSMN delegates, and the journals through social media. All coauthors of manuscripts to be discussed received an invitation to join the TweetChat and support to use Twitter, if required. A searchable hashtag \#TSSMN was created to tag the conversation and was registered with the social media analytics platform, Symplur (Symplur LLC, Los Angeles, Calif). Our group previously published a step-by-step guide on how to join a TSSMN TweetChat and provide an example of one of our prior TweetChats in the guide. $^{3}$

TweetChat and general hashtag \#TSSMN analytics were measured using Symplur. Data variables include the number of participants in the 


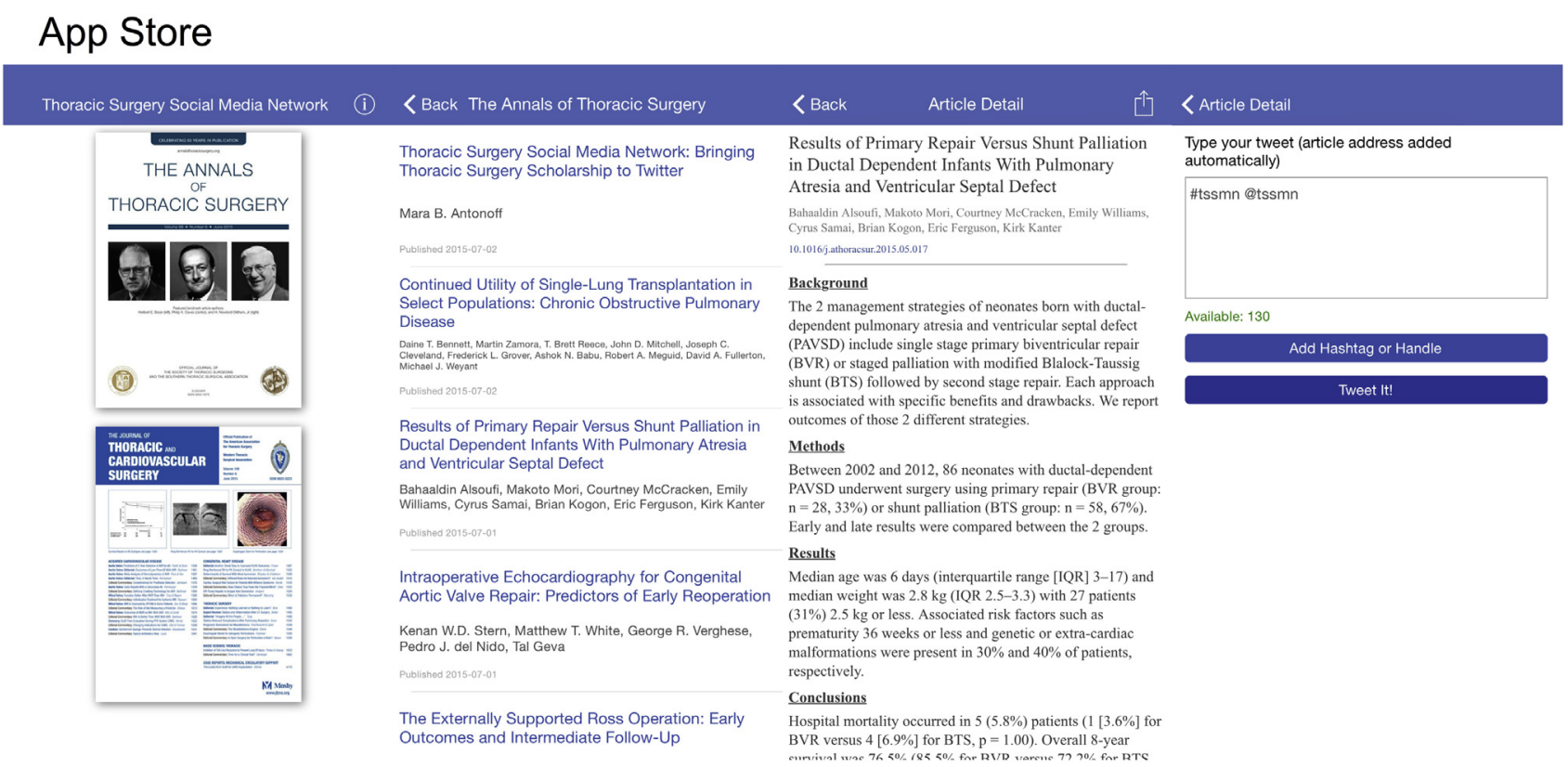

FIGURE 1. The Thoracic Surgery Social Media Network (TSSMN) Tweeter iTunes (Apple, Cuptertino, Calif) App created to streamline the process for using Twitter as a platform to expose interesting scholarly articles to followers.

TweetChat, total tweets, and impressions. Demographics of participants were provided by Symplur, and individuals were defined based on their Twitter profile and associated web links. Demographic categories are Doctor, Health Care Provider (HCP), Patient, Caregiver/Advocate, Researcher/ Academic, Journalist/Media, Individual Other Healthcare, Individual Other Non-healthcare, Organization (Org.) Provider, Org. Research/Academic, Org. Government, Org. Advocate/Support, Org. Pharma, Org. Media, Org. Other Healthcare, Org. Other Non-healthcare, Spam, Unknown. Participants may be characterized more than once (eg, a participant may be both a Doctor and a Researcher/Academic).

\section{TSSMN Tweeter iTunes App}

To streamline the process for using Twitter as a platform to expose interesting scholarly articles to followers, we developed a free mobile software application (App), the TSSMN Tweeter, available on the iTunes (Apple, Cupertino, Calif) App Store (https://itunes.apple.com/us/app/thoracicsurgery-social-media-network-tweeter/id1015160497? $1 \mathrm{~s}=1$ \&mt=8; Figure 1). The App takes advantage of the journals' RSS feeds to download the most current articles and present them to the user in a table. Tapping on a table cell will reveal the article abstract to the reader with an option to produce a tweet about it. Built in to the tweet are the \#TSSMN hashtag and the URL link that directs to the full-text article on the journal's website. The articles that are viewed on the App are tracked using a free cloud-based data store using open source software called Parse Server (http://www. back4app.com).

\section{RESULTS}

\section{TSSMN Demographics}

The Twitter handle @TSSMN has a total of 10,900 tweets, with an average of 243 tweets per month. The combined followership of all TSSMN delegates (Table 1) was 52,983 , with representation from across the world (Figure 2, A). TSSMN has 2024 followers, with a follower accumulation of 45 new followers per month, and activity at all hours of the day (Figure 2, B).
Overall, most TSSMN followers are male (64\%) and speak English (91\%), followed by Spanish (12\%), Arabic $(6 \%)$, Portuguese $(3 \%)$, and Turkish $(2 \%)$. Compared with all Twitter users, followers of TSSMN are more interested in politics $(87 \%$ vs $31 \%)$, government $(84 \%$ vs $32 \%)$, science news $(98 \%$ vs $69 \%)$, tech news $(86 \%$ vs $62 \%)$, and technology ( $84 \%$ vs $63 \%$; Figure 3$)$.

The TSSMN social media community, with communication among participants, by the \#TSSMN hashtag is shown in Figure 4 with a node-edge diagram. Biography word clouds of followers of TSSMN are shown in Figure 5, A, with the top related hashtags with \#TSSMN and topics shown in Figure 5, $B$ and $C$, respectively.

\section{TweetChats}

Hashtag \#TSSMN has a total of 17,181 total tweets, 2100 users, and 32,226,280 impressions (Figure 6), with peaks in tweeting activity of \#TSSMN corresponding to TweetChats (Table 2). Thirteen one-hour TweetChats drew a total of 489 participants, 5195 total tweets, and 17,297,708 total impressions. Figure 7 demonstrates the professional demographics of participants for the combined TweetChats. The top demographic category of TweetChat participants included individuals identified as Doctors (47\%), Org. Advocate/ Support $(11 \%)$, and Unknown $(10 \%)$ with $3 \%$ of participants characterized as patients.

The top TweetChat (Table 2) by number of tweets was "Natural History of the Aortic Root After Replacement of the Ascending Aorta" (December 13, 2017), with 670 tweets. The top TweetChat by number of impressions and participants was "Leadership of Surgical Teams in the 


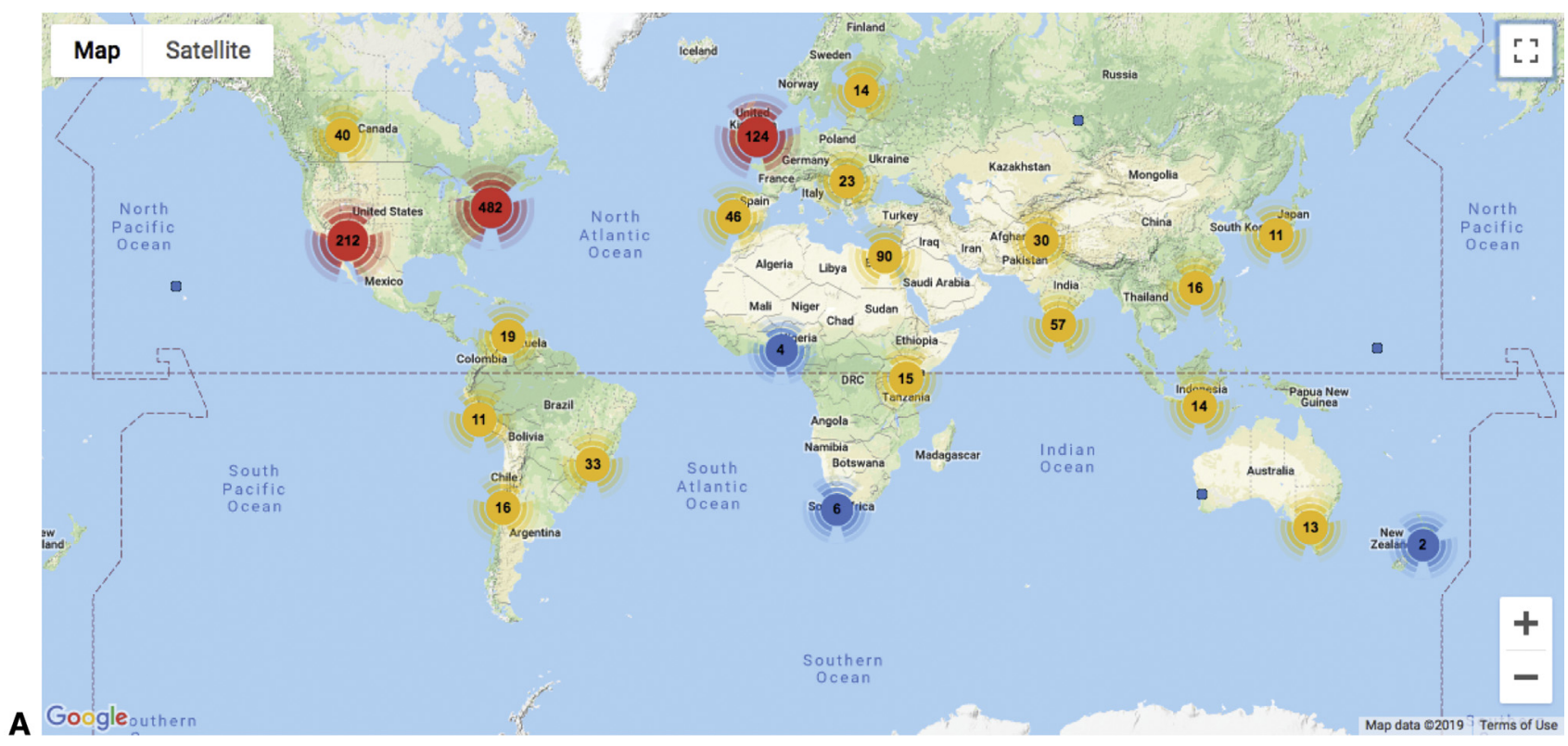

Most active hours of tssmn's followers

This chart breaks down the hourly Twitter activity of tssmn's followers.

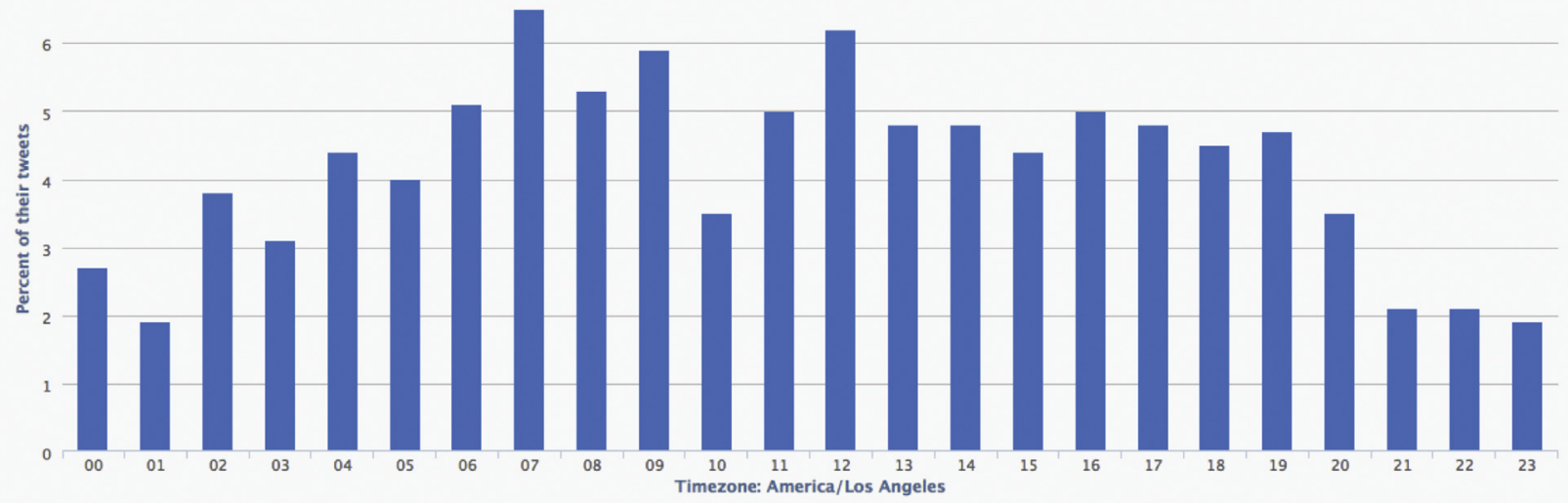

FIGURE 2. A, Geographic representation of the Thoracic Surgery Social Media Network (TSSMN) and B, Twitter activity hours of its followers.

Operating Room" (October 20, 2018), drawing 6,371,570 impressions and 72 participants. TweetChats with the lowest impressions were those held at 5 PM EST as compared with later times in the day (Table 2).

\section{TSSMN Tweeter iTunes App}

From July 2015 to February 2019, the TSSMN Tweeter iTunes App was installed 3319 times, with the majority from China $(\mathrm{n}=2643)$, followed by United States $(\mathrm{n}=390)$, Japan $(\mathrm{n}=28)$, Turkey $(\mathrm{n}=17)$, United Kingdom $(\mathrm{n}=16)$, India $(\mathrm{n}=14)$, Italy $(\mathrm{n}=13)$, Thailand $(\mathrm{n}=11)$, Brazil $(\mathrm{n}=10)$, Egypt $(\mathrm{n}=10)$, Germany $(\mathrm{n}=10)$, Russia $(\mathrm{n}=10)$, and Saudi Arabia $(\mathrm{n}=10)$. Pakistan, Mexico, Israel, Spain, Australia,
Canada, Ukraine, and Greece each accounted for fewer than 10 of the App's downloads. A total of 859 articles were viewed through the App, with 450 articles from The Annals of Thoracic Surgery and 409 from The Journal of Thoracic and Cardiovascular Surgery.

\section{COMMENT}

Social media is leading a major shift in the paradigm of how healthcare professionals connect with one another, with professional societies, journals, trainees, and of course, with patients. ${ }^{4-6}$ Social media is unique in that it has the ability to uphold the traditional goals of medical journals by disseminating peer reviewed articles to an interested audience or stakeholder. With an ever-increasing presence 


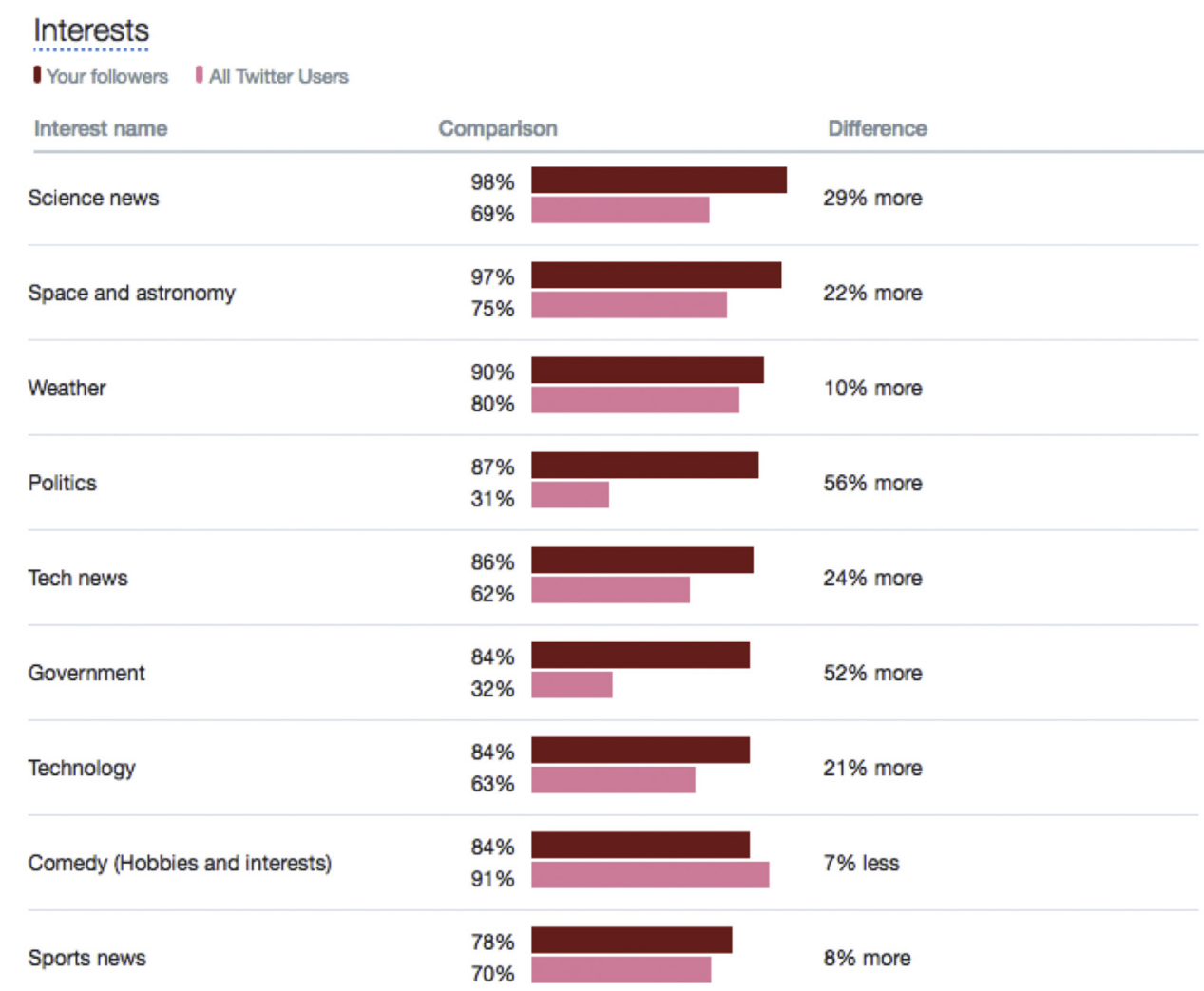

FIGURE 3. Interests of followers of the Thoracic Surgery Social Media Network compared with all other Twitter users.

of physicians and academics, social media is a strong driver of contemporary education, information dissemination, and collaboration.

TSSMN is a network created through the partnering of the 2 major cardiothoracic surgery journals with a group of diverse clinicians. We demonstrate in our preliminary evaluation of TSSMN that this online community further enhances the ability for the journals to connect with their readership and to foster a relationship that guides journal and information growth with leaders of the cardiothoracic community. Hashtag \#TSSMN had a total of 17,181 total tweets, 2100 users, and 32,226,280 impressions. Thirteen 1-hour TweetChats drew a total of 489 participants, 5195 tweets, and 17,297,708 impressions. As such, TSSMN provides a focused and readily available forum for cardiothoracic specialists.

Education is an important goal of journals, and through TSSMN, our journals have been able to facilitate educational activity. From online TweetChats to linking TSSMN with major conferences, social media not only propagates the information but also allows for a democratization of access. ${ }^{7}$ This extends beyond the article itself by including authors, experts, patients, and other stakeholders who can all contribute to the discussion around the science.
The use of articles that are freely available online also promotes access to those who would normally not have access. ${ }^{8}$ Currently, there is a global push for free online medical education as a way of allowing access to information to those who would not normally be able to by virtue of financial or geographic concerns. The partnering of TSSMN with the journals and creation of the free TSSMN Tweeter App is a way to identify and promote articles of importance that are freely available online.

Social media has been criticized for its inability to provide the robust peer reviewed information that we rely on from journals. By partnering with respected peer reviewed journals and engaging manuscript authors, readers, and the virtual community in scholarly discussion, TSSMN propagates high-quality information to the medical and lay social media community. Furthermore, by providing a channel of open, transparent, and crowd-sourced, free, open-access critical appraisal from end users, social media can arguably be the best form of postpublication peer review and may yield new thought on research, expand perspectives, and lead to collaborations. This is an important strength of TSSMN through its collaboration with clinicians and journals.

Along the lines of picking articles rather than whole issues, TSSMN members tweet about topics within their 


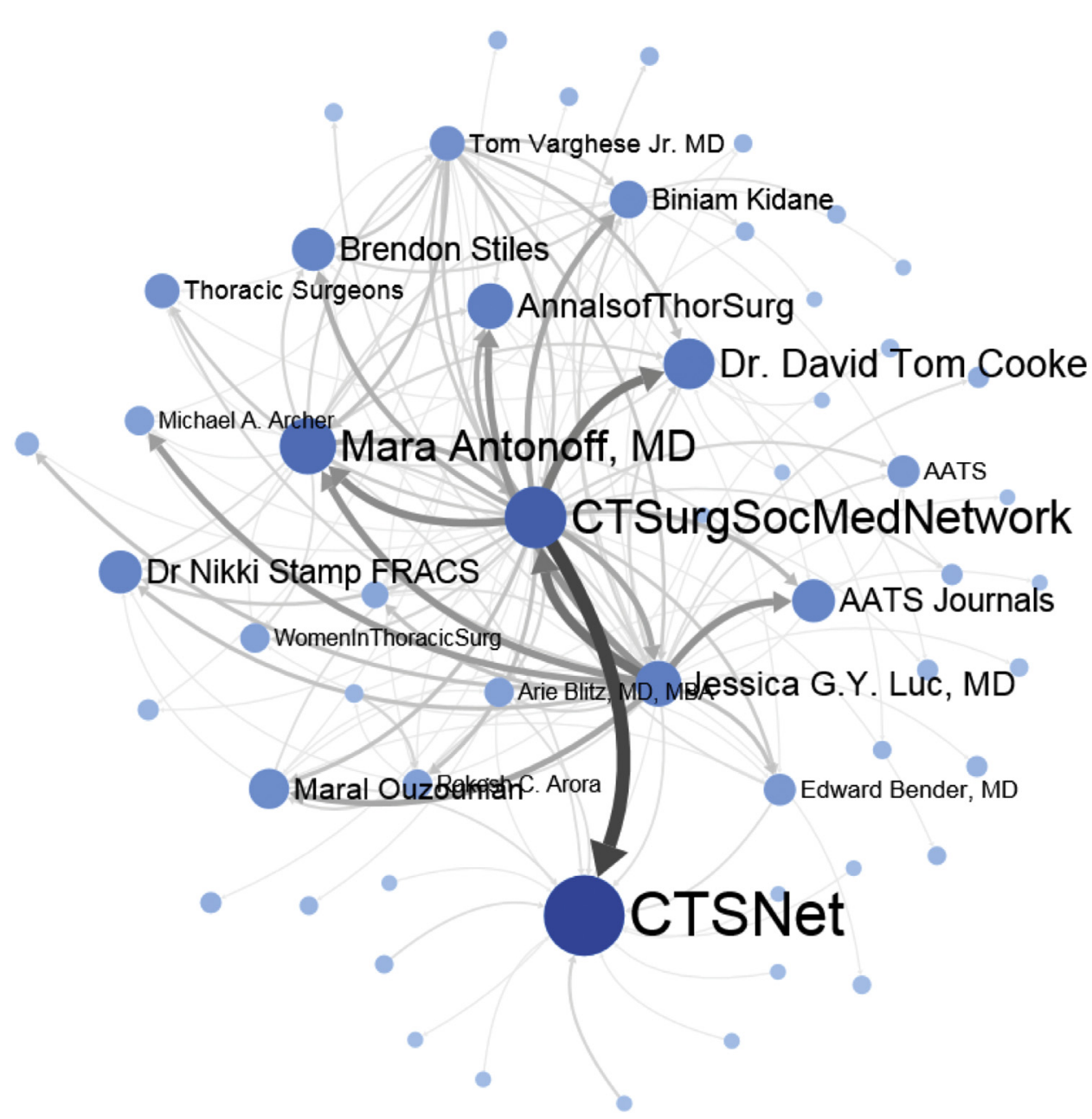

FIGURE 4. Node-edge diagram of the Thoracic Surgery Social Media Network (TSSMN) community, illustrating communication amongst participants using the \#TSSMN hashtag. Circles represent participants. The size of the circle correlates with the activity of the participant. The arrows and the size of the arrows connecting users correlate with the activity of communication. AATS, American Association for Thoracic Surgery; AnnalsofThorSurg, The Annals of Thoracic Surgery; CTSNet, Cardiothoracic Surgery Network; CTSurgSocMedNetwork, Cardiothoracic Surgery Social Media Network; Thoracic Surgeons, The Society of Thoracic Surgeons; WomenInThoracicSurg, Women in Thoracic Surgery.

area of expertise. This allows for readers to be able to categorize their reading to their area, author, or expert of choice. To streamline information discovery, TSSMN delegates can work to logically categorize articles and topics leading to increased volume of readers. TSSMN can work to both share these specialized topics and guide publishing, educational, and technological advancement to improve readership.

Further, social media attention and discussion of cardiothoracic surgery scholarship brings attention and recognition to the work of authors published in the journals. ${ }^{9}$ With social media attention being correlated with citation rates, ${ }^{10}$ we are optimistic that tweets will augment subsequent citations in the future - for the benefit of the authors, the journals, and for our specialty as a whole. A randomized prospective trial is currently underway ${ }^{11}$ with planned long-term follow-up by our group to examine whether prospective tweeting of articles through TSSMN improves article-level metrics and citations.

Given social media's permanence and widespread reach, social media is a very powerful tool that must be used responsibly. Tips on effective social media use in a surgical practice $^{3,12}$ and best practice guidelines for surgeons' social media use ${ }^{13}$ have been published by the American College of Surgeons.

\section{Lessons Learned}

Education and collaboration. In 2017, recognizing the importance of education, TSSMN added delegates representing trainees, residents, and medical students. They are uniquely placed to guide clinicians, TSSMN members, and the journals to important facets in their education. It is in this group where providing the guidance of senior clinicians and free online information where we 


\section{surgery - surgeon - thoracic - cardiac - medical - cardiothraracic - heart- rosident \\ - general - university - own - surgical - cardiovascular - research - hospital - care - health - fellow - medicine - cancer - tweets - husband - student - king - clinical \\ Two word bio cloud \\ thoracic surgeon - surgery resident - thoracic}

\section{surgery - general surgery - cardiac surgeon - cardiothoracic surgery -}

cardiac surgery - cardiothoracic surgeon - medical student - minimally invasive - lung cancer - heart surgeon - surgery

A
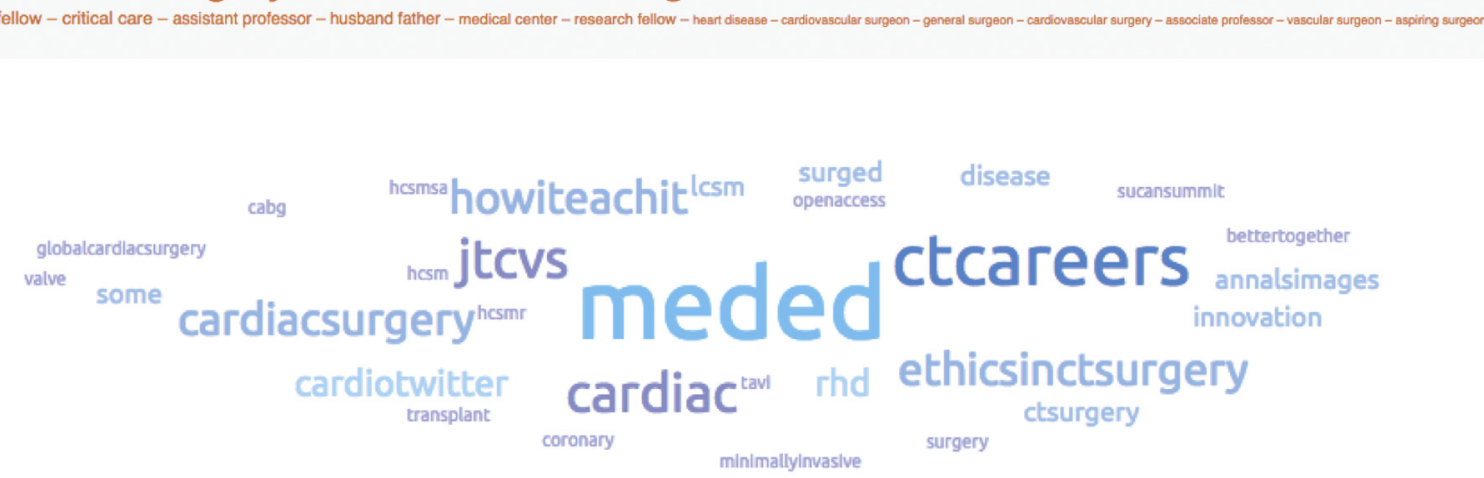

B

fellow mitral general program poster society abstracts use gibbon sublobar lung presentation health meeting outcomes sharing demonstrate sts SUrgery stage heart study fantastic popular congratulations presidentelect join article oct cancer lecture valve paper patients leaders importance recent years fibrillation risk richard januarys via thoracic resection group major cell medical clinical cover cardiac atrial surgical C longterm starts Surgeons team excited publishes proud

FIGURE 5. A, Biography word cloud of The Thoracic Surgery Social Media Network (TSSMN) followers with (B) top related hashtags and (C) topics with \#TSSMN. CABG, Coronary artery bypass grafting; HCSMSA, healthcare social media South Africa; HCSM, healthcare communications and social media; HCSMR, healthcare social media research; JTCVS, The Journal of Thoracic and Cardiovascular Surgery; LCSM, lung cancer social media network; Meded, medical education; TAVI, transcatheter aortic valve implantation; RHD, rheumatic heart disease; CTCareers, cardiothoracic surgical careers; $C T$ Surgery, cardiothoracic surgery; STS, The Society of Thoracic Surgeons; OCT, optical coherence tomography.

can see great benefits. Evidence of the feasibility of a trainee TSSMN committee has been shown through its use in the creation of a platform for cardiothoracic surgical networking and mentorship ${ }^{4-6}$ and to generate critical discussion of peer reviewed publications on trainee education to develop and implement practice-changing solutions. $^{14}$

Uniting the group in their diverse interests, trainees held joint TweetChats with TSSMN faculty, of which, one of the joint TweetChats generated the second highest impressions "Multi-arterial as compared to single-arterial grafting for coronary artery bypass grafting (CABG)" (1,377,000 impressions). Furthermore, trainees highlighted not only issues of relevance to those in cardiothoracic surgical training but also those of interest to the broader surgical community, as demonstrated by the trainee group successfully hosting the TweetChat with the most impressions and participants, "Leadership of Surgical Teams in the Operating Room" (6,371,570 impressions and 72 participants).

TweetChat timing. With such a diverse team of TSSMN delegates (Table 1) spread across 6 time zones with a maximum of 20 hours time difference and 4 countries and 12 provinces/states, finding a mutually agreeable time was challenging. TweetChats with the lowest impressions were conducted earlier in the day, at 5 PM EST (Table 2), suggesting that it is possible that many of the regular TweetChat participants were unable to join due to clinical duties.

Broadcasting TweetChats. To ensure TweetChats are appropriately broadcast to the community, we not only have had members of TSSMN tweet out an invitation but 


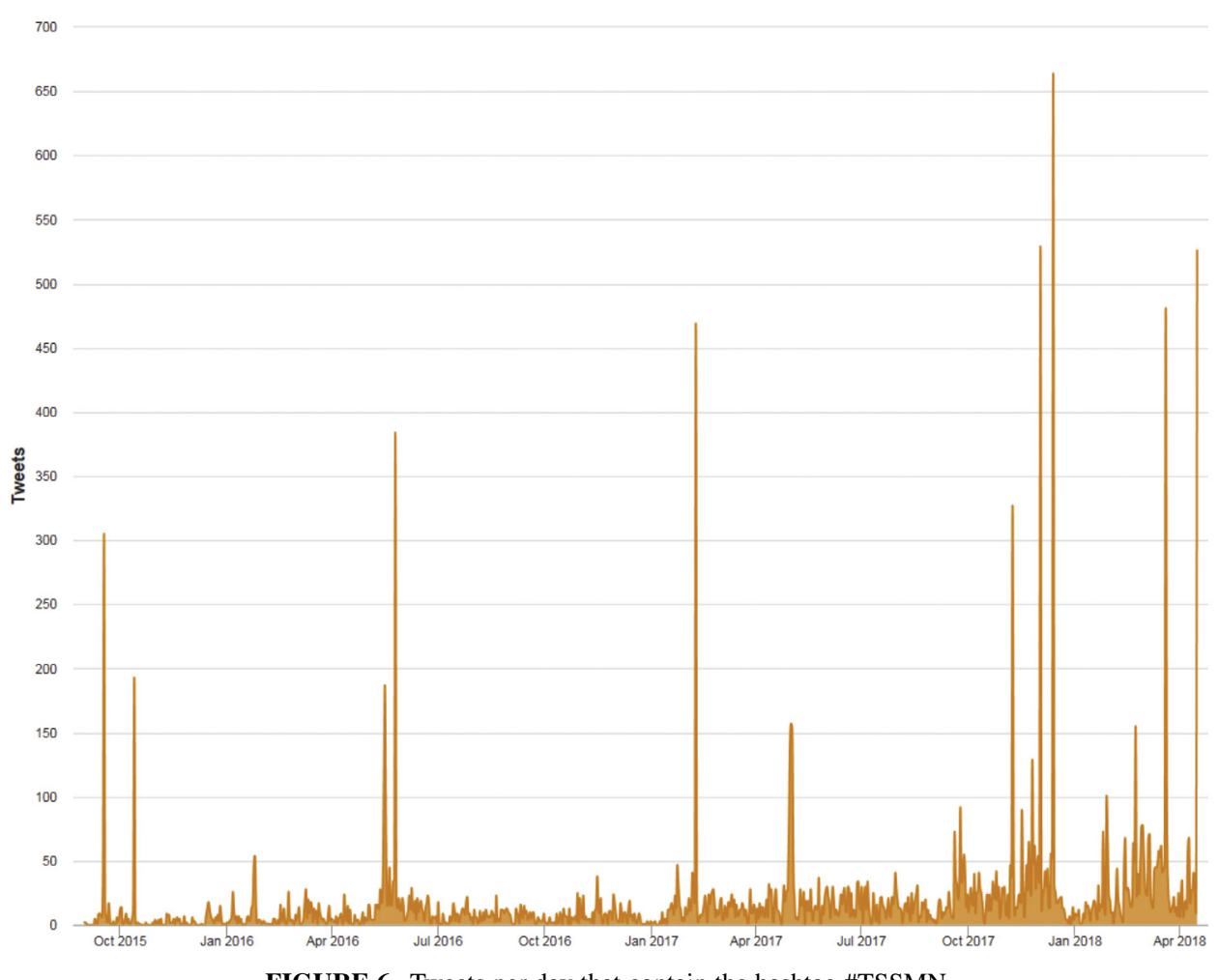

FIGURE 6. Tweets per day that contain the hashtag \#TSSMN.

also the journals and their respective societies disseminate the TweetChat ad. The TweetChat ad lists the moderator(s), TSSMN teams, discussion topic, and articles from The Annals of Thoracic Surgery and The Journal of Thoracic and Cardiovascular Surgery to be discussed. To allow for these coordinated efforts, we tend to aim for at least a 2-week notice period for people to have a chance to save the date and ensure they are available for the TweetChat.

Improving engagement. To ensure engagement and representation of authors' own voice in the discussion of their work published in the journals, we formally invited authors of articles to be discussed to the TweetChat. This not only

TABLE 2. TweetChat analytics

\begin{tabular}{|c|c|c|c|c|}
\hline TweetChat title & Date of chat (Time) & Tweets & Impressions & Participants (No.) \\
\hline SBRT in Early Stage Lung Cancer & Sep 17, 2015 (8 PM EST) & 211 & 381,519 & 22 \\
\hline TAVR & Oct 13, 2015 (8 Pм EST) & 163 & 106,234 & 8 \\
\hline AATS Annual Meeting Highlights 2016 & May 25, 2016 (9 PM EST) & 290 & $1,061,418$ & 18 \\
\hline Highlighting Key Papers From STS Annual Meeting 2017 & Feb 8, 2017 (8 PM EST) & 461 & $1,843,863$ & 44 \\
\hline Enhanced Recovery after Thoracic Surgery & Nov 8, 2017 (8 Pм EST) & 328 & $1,319,000$ & 38 \\
\hline Role of Simulation in Cardiothoracic Surgery Education & Dec 2, 2017 (6 PM EST) & 526 & $1,140,000$ & 45 \\
\hline $\begin{array}{l}\text { Natural History of the Aortic Root After Replacement of the } \\
\text { Ascending Aorta }\end{array}$ & Dec 13, 2017 (9 PM EST) & 670 & 876,747 & 60 \\
\hline Resident Assessment and Autonomy in Cardiothoracic Surgery & Mar 20, 2018 (5 PM EST) & 451 & 592,000 & 41 \\
\hline Lean Principles in Thoracic Surgery & Apr 16, 2018 (8 PM EST) & 526 & $1,009,000$ & 45 \\
\hline Non-Operative Technical Skills in Cardiothoracic Surgery & May 16, 2018 (5 PM EST) & 515 & 697,426 & 37 \\
\hline Multi-Arterial As Compared to Single-Arterial Grafting for CABG & Sep 20, 2018 (9 Рм EST) & 482 & $1,377,000$ & 39 \\
\hline Leadership of Surgical Teams in the Operating Room & Oct 20, 2018 (9 PM EST) & 362 & $6,371,570$ & 72 \\
\hline Sublobar Resections & Oct 30, 2018 (8 Pм EST) & 210 & 521,932 & 20 \\
\hline
\end{tabular}

No., Number; SBRT, stereotactic body radiotherapy; TAVR, transcatheter aortic valve replacement; AATS, American Association for Thoracic Surgery; STS, The Society of Thoracic Surgeons; $C A B G$, coronary artery bypass grafting. 

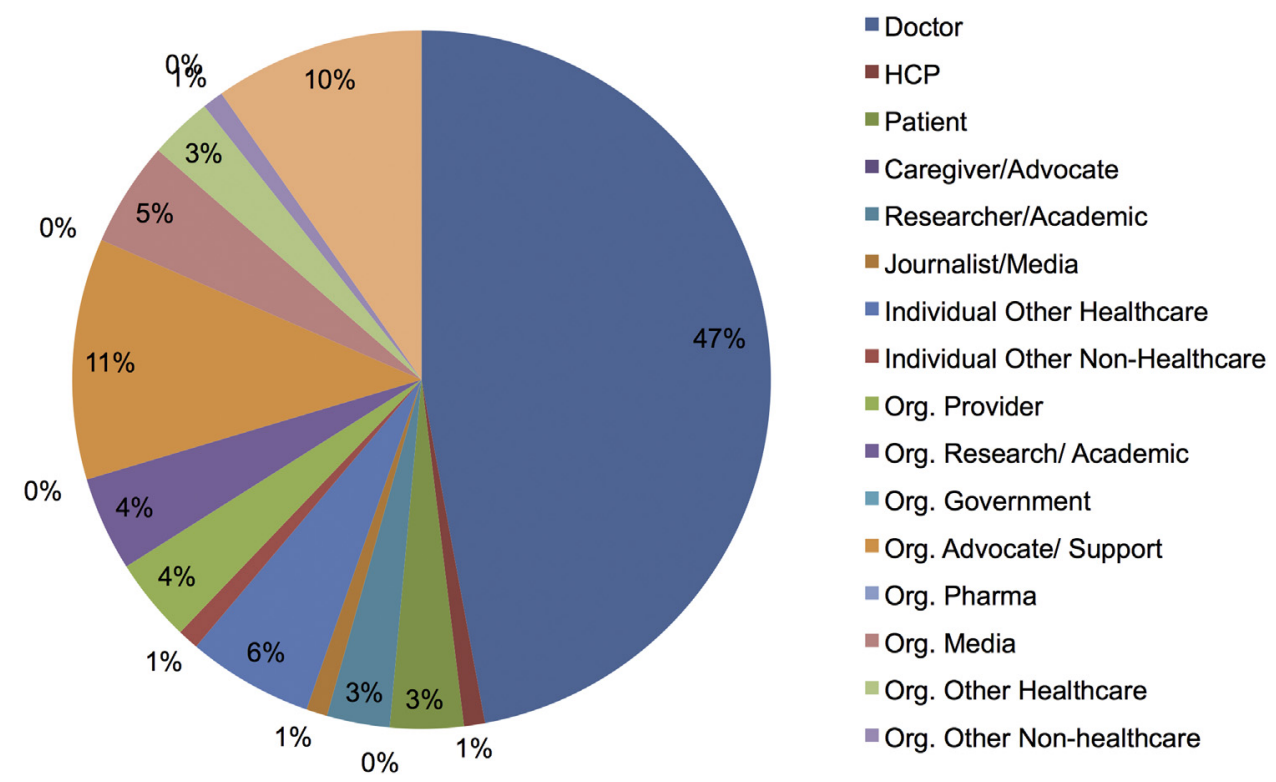

FIGURE 7. Demographics of TweetChat participants. $H C P$, Health care provider; Org, organization.

encourages more members of the cardiothoracic surgery community to join Twitter, if they have not already, but also provides a unique and valuable perspective to the work discussed with questions from colleagues and the public that can be answered by the authorship. In addition, we also made an effort to extend the invitation to colleagues outside of the realm of cardiothoracic surgery in the hopes of greater representation of a diversity of perspectives. Ultimately TweetChats are a great equalizer, allowing propagation of scientific articles to a broad audience through social networks as well as welcoming robust, constructive, real-time feedback, which is, ultimately, the optimal form of postpublication peer review, ${ }^{3}$ conducted in a novel fashion. It is interesting that most of the tweeters of articles were English speaking, whereas most of the downloads of the TSSMN Tweeter App were from China. Reasons for this may be related to unique preferences in usage of social media across the world or that users from China tweeted in English.

\section{Data Driven}

We live in an age of data and documentation. Social media efforts and outcomes are best analyzed with analytic tools, including Symplur, for TweetChat metrics including reach, impressions, and engagements. Furthermore, documentation of TweetChat content can be done with tools such as Wakelet (https://wakelet.com/) to construct a crowd-sourced collection of tweets to allow others to re-read or catch up on the TweetChat. ${ }^{3}$

\section{Limitations}

Our study is subject to a number of limitations that must be considered in the interpretation of the data. Our study represents pilot data from a limited time period. Furthermore, the study is exploratory in nature and is meant to describe the preliminary experience of a joint collaborative effort of The Annals of Thoracic Surgery and The Journal of Thoracic and Cardiovascular Surgery journals to produce a network of social media readership through TSSMN. Selection and tweeting of articles were unscheduled and left to the discretion of the individual TSSMN delegates. Although every effort was made to invite all coauthors of manuscripts scheduled for discussions at TweetChats to participate, this was not always possible given the nature of scheduling and clinical responsibilities.

In regard to the TSSMN Tweeter App, the App is currently limited to iTunes users, and expansion of the App to include Android (Google, Mountain View, Calif) users can improve user accessibility. A confounding factor in the App's utility has been the tendency for journals to display the articles' abstracts on the journal website in a nontext format, most commonly an image rendered from the journal abstract. This defeats the App's ability to parse the abstract text. Since the App user cannot read the abstract in this situation, the software becomes less efficient in tweeting about articles. A remedy would be for journals to include links to the text version of abstracts within the RSS feed.

Lastly, impressions, followers, and other social media metrics are able to demonstrate social media attention but are unable to differentiate between positive and negative attention. ${ }^{15}$ Ongoing studies to correlate social media attention with article reads, article-level metrics, citations, and journal impact factor are eagerly awaited. Furthermore, we look forward to expanding the reach of TSSMN to social media platforms beyond Twitter in the future. 


\section{Conclusion}

In these changing times, TSSMN is uniquely placed to take the Journals' readership into the future. Along with growth of actual numbers of readers and other metrics of article access, TSSMN and the Journals can shape and change our information, education, and collaboration paradigms to benefit us all. The use of TSSMN to form closer ties between the journals and clinicians puts The Annals of Thoracic Surgery and The Journal of Thoracic and Cardiovascular Surgery in prime position to create modern, technologically savvy, and thoughtful journals that is both impactful and sustainable. Ultimately, the sharing of high-quality, relevant, and easily accessible information will globalize our overarching mission to provide high-quality, up-to-date care for our patients through sound education of our clinicians. It is an exciting time to be a part of the revolution in journal-based and social media-led medical information, and TSSMN is in a unique and strong position to not only facilitate reading but also change and improve the way we communicate and understand literature.

The authors would like to acknowledge Ms Renee Bardini for her expert data collection.

\section{References}

1. Antonoff MB. Thoracic Surgery Social Media Network: bringing thoracic surgery scholarship to Twitter. J Thorac Cardiovasc Surg. 2015;150:292-3.

2. Luc JGY, Antonoff MB. Live tweet The Society of Thoracic Surgeons annual meeting: how to leverage Twitter to maximize your conference experience. Ann Thorac Surg. 2018;106:1597-601.

3. Luc JGY, Varghese Jr TK, Antonoff MB. Participating in a TweetChat: practical tips from The Thoracic Surgery Social Media Network (\#TSSMN). Ann Thorac Surg. 2019;107:e229-33.
4. Stamp NL, Luc JGY, Ouzounian M, Bhatti F, Hici TN, Antonoff MB. Social media as a tool to rewrite the narrative for women in cardiothoracic surgery. Interact Cardiovasc Thorac Surg. 2019;28:831-7.

5. Luc JGY, Stamp NL, Antonoff MB. Social media as a means of networking and mentorship: role for women in cardiothoracic surgery. Semin Thorac Cardiovasc Surg. 2018;30:487-95.

6. Luc JGY, Stamp NL, Antonoff MB. Social media in the mentorship and networking of physicians: important role for women in surgical specialties. Am J Surg. 2018;215:752-60.

7. Luc JGY, Antonoff MB. Active learning in medical education: application to the training of surgeons. J Med Educ Curric Dev. 2016; 3. JMECD.S18929.

8. Chan T, Trueger NS, Roland D, Thoma B. Evidence-based medicine in the era of social media: scholarly engagement through participation and online interaction. CJEM. 2018;20:3-8.

9. Hawkins CM, Hillman BJ, Carlos RC, Rawson JV, Haines R, Duszak Jr R. The impact of social media on readership of a peer-reviewed medical journal. J Am Coll Radiol. 2014;11:1038-43.

10. Lamb CT, Gilbert SL, Ford AT. Tweet success? Scientific communication correlates with increased citations in Ecology and Conservation. PeerJ. 2018; 6:e4564.

11. Luc JGY, Archer MA, Arora RC, Bender EM, Blitz A, Cooke DT, et al. Social media improves cardiothoracic surgery literature dissemination: results of a randomized trial. Ann Thorac Surg. June 17, 2019 [Epub ahead of print].

12. Antonoff MB. Using social media effectively in a surgical practice. J Thorac Cardiovasc Surg. 2016;151:322-6.

13. Logghe HJ, Boeck MA, Gusani NJ, Hardaway JC, Hughes KA, Mouawad NJ, et al. Best practices for surgeons' social media use: statement of the Resident and Associate Society of the American College of Surgeons. J Am Coll Surg. 2018;226:317-27.

14. Ni hlci T, Archer M, Harrington C, Luc JGY, Antonoff MB. The Trainee Thoracic Surgery Social Media Network: early experience with TweetChatbased journal clubs. Ann Thorac Surg. May 28, 2019 [Epub ahead of print].

15. Trueger N, Thoma B, Hsu CH, Sullivan D, Peters L, Lin M. The Altmetric score: a new measure for article-level dissemination and impact. Ann Emerg Med. 2015; 66:549-53.

Key Words: Social media, health care community, communication, collaboration, education 